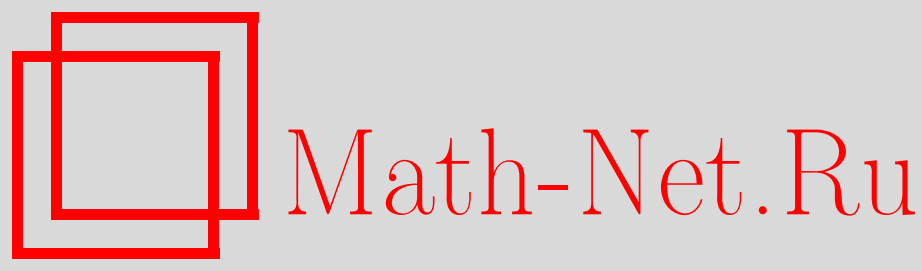

А. О. Савченко, Функции, ортогональные к многочленам, и их применение в осесимметричных задачах физики, ТМФ, 2014, том 179, номер 2, 225-241

DOI: https://doi.org/10.4213/tmf8567

Использование Общероссийского математического портала Math-Net.Ru подразумевает, что вы прочитали и согласны с пользовательским соглашением http://www.mathnet.ru/rus/agreement

Параметры загрузки:

IP : 3.85 .183 .62

26 апреля 2023 г., 09:22:05

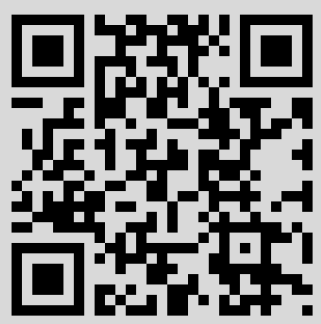




\title{
ФУНКЦИИ, ОРТОГОНАЛЬНЫЕ К МНОГОЧЛЕНАМ, И ИХ ПРИМЕНЕНИЕ В ОСЕСИММЕТРИЧНЫХ ЗАДАЧАХ ФИЗИКИ
}

\begin{abstract}
Исследованы свойства функциональных семейств, которые состоят из счетного количества элементов $A_{n}$ таких, что каждая функция $A_{n}$ ортогональна всем многочленам степени меньше $n$. Предложен эффективный метод решения интегральных уравнений Фредгольма первого рода с ядрами, являющимися производящими функциями для таких функций. Изучены интегральные уравнения, использующиеся для решения некоторых осесимметричных задач физики. Доказано, что их ядра являются производящими функциями, которые производят функции из исследуемых семейств, и найден явный вид этих фукнций. Данное обстоятельство позволяет определить элементы матриц систем линейных уравнений, связанных с интегральными уравнениями для рассмотренных задач физики.
\end{abstract}

Ключевые слова: производящие функции, функции, ортогональные к многочленам, интегральные уравнения Фредгольма первого рода, осесимметричное тело.

DOI: $10.4231 / \operatorname{tmf} 8567$

\section{1. ВВЕДЕНИЕ}

Эффективные методы решения многих задач физики основываются на использовании присущих им особенностей. В настоящей работе такой особенностью является наличие осесимметричного тела, расположенного в среде, обладающей свойством симметрии; ось тела направлена вдоль оси симметрии среды. Такой средой может быть электрическое или магнитное поле, а также движущийся поток идеальной стационарной струи. Задача состоит в определении физических характеристик явлений на поверхности тела, возникающих под воздействием внешней среды, например поверхностных зарядов, поверхностных токов или скорости жидкости вблизи поверхности обтекаемого тела. Искомые физические характеристики могут быть найдены путем решения одномерных интегральных уравнений Фредгольма первого рода. Такой подход к решению каждой из рассмотренных задач был предложен

* Институт вычислительной математики и математической геофизики СО РАН, Новосибирск, Россия. E-mail: savch@ommfao1.sscc.ru 
в работах [1]-[3]. В указанных работах искомые решения являлись многочленами степени $N$, если правые части уравнений являлись многочленами той же степени. Для нахождения коэффициентов этих многочленов достаточно было решить систему линейных уравнений с треугольной матрицей размера $(N+1) \times(N+1)$. Однако способ определения элементов матрицы линейных уравнений был достаточно трудоемким и потому малопригодным с практической точки зрения уже для матриц небольших размеров.

В настоящей работе предложен общий метод решения интегральных уравнений Фредгольма первого рода с ядрами, являющимися производящими функциями, которые производят функции, ортогональные к многочленам меньшей степени (определение множества таких функций приведено в начале раздела 2). В разделе 2 исследуются свойства этих функций. Установлена связь между моментами производящей функции и ее разложением, предложен способ определения элементов матрицы линейных уравнений для искомых коэффициентов многочлена решения и исследованы ее свойства. В разделе 3 исследуются свойства нескольких семейств функций, непрерывно зависящих от параметра, которые используются при решении рассматриваемых далее физических задач. Доказано, что производимые ими функции ортогональны к многочленам меньшей степени, и найден их явный вид. Эти функции использованы в дальнейшем для нахождения элементов матрицы линейных уравнений. В разделе 4 для каждой рассмотренной физической задачи приведен метод решения и конкретизирован вид системы линейных уравнений с учетом результатов, полученных в двух предыдущих разделах.

\section{2. СВОЙСТВА ФУНКЦИЙ, ОРТОГОНАЛЬНЫХ К МНОГОЧЛЕНАМ МЕНЬШЕЙ СТЕПЕНИ}

ОПРЕДЕЛЕНИЕ 1. Будем называть функции $A_{0}(x), A_{1}(x), \ldots, A_{n}(x), \ldots$ ортогональными $\kappa$ многочленам меньшей степени, если для любого $n=0,1, \ldots$ имеет место ортогональность $A_{n}(x) \perp x^{k}$ для всех целых неотрицательных $k<n$.

Мы будем рассматривать функции, ортогональные в смысле скалярного произведения в $L_{2}[a, b]$ с единичным весом. Функциями, ортогональными к многочленам меньшей степени, являются, например, многочлены Лежандра, а также другие многочлены Якоби, помноженные на весовые функции. Однако если весовые функции не равны единице, то функции, ортогональные к многочленам меньшей степени, не ортогональны друг к другу.

Нетрудно показать, используя ортогонализацию Грама-Шмидта в “обратном порядке" от последней функции к первой, что для любого конечного множества функций $A_{n}(x) \in L_{2}[a, b], n=0,1, \ldots, N$, ортогональных к многочленам меньшей степени, существуют ортогональные функции $B_{0}(x), B_{1}(x), \ldots, B_{N}(x)$, принадлежащие линейной оболочке функций $A_{0}(x), A_{1}(x), \ldots, A_{N}(x)$ и ортогональные к многочленам меньшей степени.

Для нахождения функций $A_{n}(x)$, ортогональных к многочленам меньшей степени, будем использовать их производящие функции, т. е. такие функции $G(x, s)$, для 
которых выполнено равенство

$$
G(x, s)=\sum_{n=0}^{\infty} A_{n}(x) s^{n} .
$$

Из этого равенства можно выразить любую производимую функцию через производящую функцию по формуле

$$
A_{n}(x)=\left.\frac{1}{n !} \frac{\partial^{n} G(x, s)}{\partial s^{n}}\right|_{s=0} .
$$

ОПРЕДЕЛЕНИЕ 2. Назовем производящую функцию $G(x, s)$, заданную формулой (1), порождающей функцией, если производимые ею функции $A_{n}(x)$ ортого-

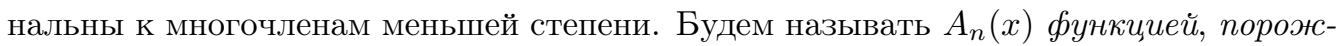
даемой функиией $G(x, s)$.

Пусть порождаемые функции ортогональны к многочленам меньшей степени на отрезке $[a, b]$. Далее обозначим через $p_{k}(s)$ произвольный многочлен степени $k$.

ТЕОрема 1. Производящая функиия $G(x, s)$ является порождающей функиией тогда и только тогда, когда выполнено равенство

$$
\int_{a}^{b} G(x, s) x^{k} d x=p_{k}(s)
$$

для любого целого неотрицательного значения $k$.

ДокАзАТЕЛЬСтво. Пусть функция $G(x, s)$ является порождающей. Тогда, поскольку все порождаемые функции $A_{n}(x)$ ортогональны к $x^{k}$ при $n>k$,

$$
\int_{a}^{b} G(x, s) x^{k} d x=\sum_{n=0}^{\infty} s^{n} \int_{a}^{b} A_{n}(x) x^{k} d x=\sum_{n=0}^{k} s^{n} \int_{a}^{b} A_{n}(x) x^{k} d x=p_{k}(s) .
$$

В другую сторону: пусть равенство (3) выполнено для производящей функции $G(x, s)$ при любых целых неотрицательных значениях $k$. Тогда с учетом определения (1) получим, что

$$
\sum_{n=k+1}^{\infty} s^{n} \int_{a}^{b} A_{n}(x) x^{k} d x=0
$$

для любых значений $s$ из интервала сходимости ряда (1), а это может иметь место только тогда, когда все коэффициенты ряда (4) равны нулю, т. е.

$$
\int_{a}^{b} A_{n}(x) x^{k} d x=0 \quad \text { при } \quad n>k,
$$

следовательно, производящая функция $G(x, s)$ является порождающей. Теорема доказана.

СлЕДСТВИЕ 1. Если функиия $G(x, s)$ является порождающей, то функция

$$
\left(\alpha_{1}+\alpha_{2} x\right) \frac{\partial G(x, s)}{\partial s}
$$

также будет порождающей для любых значений параметров $\alpha_{1} u \alpha_{2}$. 
ДокАЗАТЕЛЬСтво. Так как для порождающей функции справедливо соотношение (3), мы имеем

$$
\int_{a}^{b} \frac{\partial G(x, s)}{\partial s} x^{k} d x=p_{k-1}(s)
$$

Тогда из теоремы 1 следует, что функции $\frac{\partial G(x, s)}{\partial s}$ и $x \frac{\partial G(x, s)}{\partial s}$ будут порождающими. Очевидно, что линейная комбинация этих функций также будет порождающей функцией.

Функции $A_{n}^{(1)}(x)$, порождаемые линейной комбинацией (5), нетрудно выразить через функции, порождаемые функцией $G(x, s)$. Действительно,

$$
\left(\alpha_{1}+\alpha_{2} x\right) \frac{\partial G(x, s)}{\partial s}=\left(\alpha_{1}+\alpha_{2} x\right) \sum_{n=1}^{\infty} n A_{n}(x) s^{n-1}=\left(\alpha_{1}+\alpha_{2} x\right) \sum_{n=0}^{\infty}(n+1) A_{n+1}(x) s^{n},
$$

отсюда

$$
A_{n}^{(1)}(x)=\left(\alpha_{1}+\alpha_{2} x\right)(n+1) A_{n+1}(x) .
$$

Заметим, что произведение $k$-й производной по переменной $s$ от порождающей функции и многочлена степени, не превышающей $k$, также является порождающей функцией.

Теорема 2. Пусть функиия $G(x, s)$ является порождающей на отрезке $[a, b]$, а функция $f(s)$ - многочлен степени $N$,

$$
f(s)=\sum_{n=0}^{N} b_{n} s^{n}
$$

Тогда решением интегрального уравнения

$$
\int_{a}^{b} G(x, s) u(x) d x=f(s)
$$

является многочлен $u(x)=\sum_{k=0}^{N} c_{k} x^{k}$ степени $N$, коэфбициенты которого удовлетворяют системе линейных уравнений

$$
\mathbf{F c}=\mathbf{b},
$$

əде $\mathbf{c}=\left(c_{0}, c_{1}, \ldots, c_{N}\right)^{\mathrm{T}}, \mathbf{b}=\left(b_{0}, b_{1}, \ldots, b_{N}\right)^{\mathrm{T}}, \mathbf{F}-$ матрица с элементами

$$
F_{n k}=\int_{a}^{b} A_{n-1}(x) x^{k-1} d x, \quad n, k=1,2, \ldots, N+1,
$$

а функиии $A_{n-1}(x), n=1,2, \ldots, N+1$, являются порождаемыми функиией $G(x, s)$.

ДокАзАтЕльСтво. Докажем первое утверждение. Любой многочлен $f(s)$ степени $N$ можно единственным способом представить в виде [4]

$$
f(s)=\sum_{k=0}^{N} c_{k} p_{k}(s),
$$


где $p_{0}(s), p_{1}(s), \ldots p_{N}(x)$ - заданная система многочленов такая, что многочлен $p_{k}$ имеет степень $k$ для каждого $k=0,1, \ldots, N$. Отсюда с учетом (3) и (12) следует, что

$$
f(s)=\int_{a}^{b} G(x, s)\left(\sum_{k=0}^{N} c_{k} x^{k}\right) d x .
$$

Таким образом, $u(x)=\sum_{k=0}^{N} c_{k} x^{k}$ является решением интегрального уравнения (9).

Поскольку функция $G(x, s)$ порождающая, для нее справедливо разложение (1). Тогда с учетом равенства (8) уравнение (13) можно записать в виде

$$
\int_{a}^{b} \sum_{n=0}^{\infty} A_{n}(x) s^{n} \sum_{k=0}^{N} c_{k} x^{k} d x=\sum_{n=0}^{\infty} s^{n} \sum_{k=0}^{N} c_{k} \int_{a}^{b} A_{n}(x) x^{k} d x=\sum_{n=0}^{N} b_{n} s^{n} .
$$

Данное уравнение справедливо для всех значений $s$ из интервала сходимости ряда (1), отсюда получаем следующую систему линейных уравнений для определения искомых коэффициентов $c_{k}, k=0,1, \ldots, N$ :

$$
\sum_{k=0}^{N} c_{k} \int_{a}^{b} A_{n}(x) x^{k} d x=b_{n}, \quad n=0,1, \ldots, N .
$$

Эту систему можно записать в матричном виде (10).

СЛЕДСТВиЕ 2. Матрица $\mathbf{F}$ является верхнетреуголъной.

ДокАЗАТЕЛЬСтво. Функции $A_{n}(x)$ являются порождаемыми, таким образом, по определению $A_{n}(x) \perp x^{k}$ при $n>k$. Отсюда с учетом (11) мы получаем утверждение следствия.

СлеДСТВИЕ 3. Если $a=-b$, а четность функции $A_{n}(x)$ совпадает с четностъю индекса $n$ для всех $n=0,1, \ldots, N$, то элементы матрицы $\mathbf{F}$, стоящие в нечетных верхних диагоналях, равны нулю, т.е. $F_{n k}=0$, если $n+k$ - нечетное число.

ДокАзАТЕЛЬство следует из формулы (11).

\section{3. СЕМЕЙСТВА ФУНКЦИЙ, ОРТОГОНАЛЬНЫХ К МНОГОЧЛЕНАМ МЕНЬШЕЙ СТЕПЕНИ И НЕПРЕРЫВНО ЗАВИСЯЩИХ ОТ ПАРАМЕТРА}

Рассмотрим несколько семейств функций, ортогональных к многочленам меньшей степени на отрезке конечной длины и непрерывно зависящих от параметра. Одно из таких двухпараметрических семейств функций хорошо известно. Это многочлены Якоби, помноженные на весовые функции вида $(1-x)^{\alpha}(1+x)^{\beta}$, где $\alpha>-1$ и $\beta>-1$. В настоящей работе мы будем рассматривать другие семейства таких функций, имеющие приложения в осесимметричных задачах физики.

3.1. Будем рассматривать производящую функцию вида

$$
G_{0}(x, s)=\frac{1}{\sqrt{(x-s)^{2}+\gamma^{2}\left(1-x^{2}\right)}}, \quad \gamma \in(0,1], \quad s \in(-1,1) .
$$

Заметим, что при $\gamma=1$ эта функция совпадает с производящей функцией для многочленов Лежандра. 
ТЕОРема 3. Производящая функиия $G_{0}(x, s)$ является порождающей на отрезке $[-1,1]$.

ДокАзАтельство. Докажем, что

$$
\int_{-1}^{1} G_{0}(x, s) x^{n} d x=p_{n}(s)
$$

где через $p_{n}(s)$ обозначим, как и ранее, некоторый многочлен степени $n$. Доказательство будем проводить по индукции. Положим

$$
R=R(x, s)=\sqrt{(x-s)^{2}+\gamma^{2}\left(1-x^{2}\right)}
$$

При $n=0$ имеем

$$
\int \frac{d x}{R}=\frac{1}{\sqrt{1-\gamma^{2}}} \ln \left(\sqrt{\left(1-\gamma^{2}\right) R^{2}}+\left(1-\gamma^{2}\right) x-2 s\right),
$$

откуда

$$
\int_{-1}^{1} \frac{d x}{R}=\frac{1}{\sqrt{1-\gamma^{2}}} \ln \frac{1+\sqrt{1-\gamma^{2}}}{1-\sqrt{1-\gamma^{2}}}
$$

и интеграл не зависит от значения переменной $s$.

При $n=1$ имеем

$$
\int \frac{x d x}{R}=\frac{1}{1-\gamma^{2}}\left(R+s \int \frac{d x}{R}\right)
$$

Нетрудно заметить, что тогда определенный интеграл $\int_{-1}^{1}(x / R) d x$ будет линейной функцией от переменной $s$.

Пусть равенство (17) имеет место при $n=k-1$; докажем его справедливость при $n=k$. Воспользовавшись интегралом из справочника [5], получим

$\int_{-1}^{1} \frac{x^{k} d x}{R}=\frac{1}{k\left(1-\gamma^{2}\right)}\left(\left.x^{k-1} R\right|_{-1} ^{1}+(2 k-1) s \int_{-1}^{1} \frac{x^{k-1} d x}{R}-(k-1)\left(\gamma^{2}+s^{2}\right) \int_{-1}^{1} \frac{x^{k-2} d x}{R}\right)$.

Поскольку по индукционному предположению первый и второй интегралы в правой части равенства являются соответственно многочленами $(k-1)$-й и $(k-2)$-й степени по переменной $s$, интеграл в левой части равенства будет многочленом степени $k$ по переменной $s$. Таким образом, равенство (17) справедливо для любых значений $n$, и по теореме 1 функция $G_{0}(x, s)$ является порождающей. Теорема доказана.

Найдем явный вид функций $H_{n}(x)$, порождаемых функцией $G_{0}(x, s)$. Введем обозначение

$$
Q=Q(x)=R(x, 0)=\sqrt{\left(1-\gamma^{2}\right) x^{2}+\gamma^{2}} .
$$

ТеОРема 4. Функиии $H_{n}(x)$, порождаемые функцией $G_{0}(x, s)$, имеют вид

$$
H_{n}(x)=\frac{1}{Q^{n+1}} P_{n}\left(\frac{x}{Q}\right),
$$

где $P_{n}(t)$ - многочлены Лежандра степени $n$. 
ДокАзАТЕЛЬство. Хорошо известно [4], что для многочленов Лежандра порождающей является функция

$$
\frac{1}{\sqrt{1-2 s x+s^{2}}}=\sum_{n=0}^{\infty} P_{n}(x) s^{n} .
$$

Отсюда

$$
\left(1-2 \frac{s}{Q} \frac{x}{Q}+\left(\frac{s}{Q}\right)^{2}\right)^{-1 / 2}=\frac{Q}{\sqrt{Q^{2}-2 s x+s^{2}}}=\sum_{n=0}^{\infty} P_{n}\left(\frac{x}{Q}\right)\left(\frac{s}{Q}\right)^{n} .
$$

Последнее равенство в (21) имеет место при

$$
\frac{|x|}{Q} \leqslant 1, \quad \frac{|s|}{Q}<1 .
$$

Для $Q$, заданного в (19), первое неравенство в (22) справедливо при $|x| \leqslant 1$, а второе неравенство будет справедливым для всех $x$ при выполнении условий $|s|<\gamma \leqslant 1$. Из формул (19) и (21) следует, что

$$
\frac{1}{\sqrt{(x-s)^{2}+\gamma^{2}\left(1-x^{2}\right)}}=\sum_{n=0}^{\infty} \frac{1}{Q^{n+1}} P_{n}\left(\frac{x}{Q}\right) s^{n}
$$

таким образом, функции, порождаемые $G_{0}(x, s)$, имеют вид $(20)$.

СлЕДСТвИЕ 4. Функиии $H_{n}(x)$ удовлетворяют рекуррентному соотношению

$$
(n+1) Q^{2} H_{n+1}(x)=(2 n+1) x H_{n}(x)-n H_{n-1}(x) .
$$

ДоказАтельство. Равенство (23) следует из рекуррентного соотношения для многочленов Лежандра

$$
(n+1) P_{n+1}(x)=(2 n+1) x P_{n}(x)-n P_{n-1}(x)
$$

и формулы $(20)$.

СлеДСТВИЕ 5. Функции $H_{2 k}(x)$ четные, а функции $H_{2 k+1}(x)$ нечетные для любых целых неотрицательных значений $k$.

ДокАЗАтЕЛьство. Из формулы (20) следует, что $H_{0}(x)=1 / Q$ и $H_{1}(x)=x / Q^{3}$. Доказательство утверждения получается применением математической индукции к рекуррентной формуле (23).

СлЕДСТвИЕ 6. Для производной функиии $H_{n}(x)$ справедлива формула

$$
H_{n}^{\prime}(x)=\frac{n+1}{x^{2}-1}\left(H_{n+1}(x)-x H_{n}(x)\right) .
$$

ДокАЗАТЕЛЬСтво. Положим $t=x / Q$. Взяв производную от обеих частей равенства (20), получим

$$
\frac{d H_{n}(x)}{d x}=\frac{d}{d x}\left(\frac{1}{Q^{n+1}}\right) P_{n}(t)+\frac{1}{Q^{n+1}} \frac{d P_{n}(t)}{d t} \frac{d t}{d x} .
$$


Поскольку для многочленов Лежандра справедлива формула [4]

$$
\frac{d P_{n}(t)}{d t}=\frac{n}{t^{2}-1}\left(t P_{n}(t)-P_{n-1}(t)\right)
$$

и

$$
\frac{d t}{d x}=\frac{1}{Q}-\frac{\left(1-\gamma^{2}\right) x^{2}}{Q^{3}}, \quad \frac{d}{d x}\left(\frac{1}{Q^{n+1}}\right)=-\frac{(n+1)\left(1-\gamma^{2}\right) x}{Q^{n+3}},
$$

из (25) получим

$$
H_{n}^{\prime}(x)=\frac{1}{Q^{2}\left(x^{2}-1\right)}\left[x\left(2 n+1-(n+1) Q^{2}\right) H_{n}(x)-n H_{n-1}(x)\right] .
$$

Выразив последнее слагаемое в правой части равенства по рекуррентной формуле (23), получим формулу (24).

3.2. Рассмотрим еще одно семейство функций, непрерывно зависящих от параметра $\gamma$ и ортогональных к многочленам меньшей степени. Прежде всего, отметим, что производная по переменной $s$ от порождающей функции тоже будет порождающей функцией (см. следствие 1). В частности, функция

$$
\frac{\partial G_{0}(x, s)}{\partial s}=\frac{x-s}{\left[(x-s)^{2}+\gamma^{2}\left(1-x^{2}\right)\right]^{3 / 2}}=\frac{x-s}{R^{3}}
$$

является порождающей. Рассмотрим другие порождающие функции.

Теорема 5. Функиия $\left(1-s^{2}\right) / R^{3}$ является порождающей функиией.

ДокАЗАТЕЛЬСтво. Непосредственным интегрированием получим

$$
\int_{-1}^{1} \frac{1-s^{2}}{R^{3}} d x=\frac{2}{\gamma^{2}}
$$

Так как функция $G_{0}(x, s)$ порождающая, из теоремы 1 следует, что

$$
\int_{-1}^{1} G_{0}(x, s) d x=\text { const }, \quad \int_{-1}^{1} \frac{\partial G_{0}(x, s)}{\partial s} d x=0 .
$$

Отсюда с учетом (26) получаем

$$
\int_{-1}^{1} \frac{1}{R^{3}} x d x=s \int_{-1}^{1} \frac{d x}{R^{3}}=\frac{2 s}{\gamma^{2}\left(1-s^{2}\right)}, \quad \int_{-1}^{1} \frac{1-s^{2}}{R^{3}} x d x=\frac{2 s}{\gamma^{2}} .
$$

Предположим, что для некоторого натурального $n$ имеет место равенство

$$
\int_{-1}^{1} \frac{1-s^{2}}{R^{3}} x^{n} d x=p_{n}(s)
$$

и докажем, что будет выполнено равенство

$$
\int_{-1}^{1} \frac{1-s^{2}}{R^{3}} x^{n+1} d x=p_{n+1}(s) .
$$


Поскольку функция $G_{0}(x, s)$ порождающая, из соотношений $(6)$ и $(26)$ следует, что

$$
\int_{-1}^{1} \frac{x-s}{R^{3}} x^{n} d x=p_{n-1}(s) .
$$

Отсюда

$$
\int_{-1}^{1} \frac{x^{n+1}}{R^{3}} d x=s \int_{-1}^{1} \frac{x^{n}}{R^{3}} d x+p_{n-1}(s) .
$$

В свою очередь из (27) получим

$$
\int_{-1}^{1} \frac{x^{n}}{R^{3}} d x=\frac{p_{n}(s)}{1-s^{2}}
$$

тогда

$$
\int_{-1}^{1} \frac{x^{n+1}}{R^{3}} d x=s \frac{p_{n}(s)}{1-s^{2}}+p_{n-1}(s),
$$

откуда и следует равенство (28). Таким образом, равенство (27) выполнено для любых значений $n$, и из теоремы 1 следует, что функция $\left(1-s^{2}\right) / R^{3}$ порождающая.

СлЕДСТВИЕ 7. Функции вида

$$
\frac{(x-s)(a x+b s+c)+d\left(1-s^{2}\right)}{R^{3}},
$$

где $a, b, c, d$ - произвольные константы, являются порождающими.

ДокАЗАТЕЛЬСТво. Если функция $G(x, s)$ порождающая, то в силу следствия 1 порождающими будут также функции вида $\left(\alpha_{1}+\alpha_{2} x\right) \frac{\partial G(x, s)}{\partial s}$. Из равенства (6) следует, что порождающей будет и функция $s \frac{\partial G(x, s)}{\partial s}$. Из теоремы 1 следует, что линейная комбинация порождающих функций также будет порождающей функцией. Положив $G(x, s)=G_{0}(x, s)$, получим с учетом $(26)$ и теоремы 5 требуемое утверждение.

Рассмотрим важный частный случай функции вида (29). Пусть $a=b=-1$, $c=0, d=1$. Тогда получим порождающую функцию

$$
G_{1}(x, s)=\frac{1-x^{2}}{R^{3}},
$$

числитель которой не зависит от переменной $s$.

ТЕорема 6. Функиии $\widetilde{H}_{n}(x)$, порождаемые функиией $G_{1}(x, s)$, имеют вид

$$
\widetilde{H}_{n}(x)=\frac{n+1}{\gamma^{2}}\left(H_{n}(x)-x H_{n+1}(x)\right) .
$$

ДокАзАТЕЛЬСтво. Найдем функции, производимые производящей (не порождающей) функцией $1 / R^{3}$. Так как функция $G_{0}(x, s)$ порождает функции $H_{n}(x)$, имеем

$$
\frac{\partial G_{0}(x, s)}{\partial x}=\frac{s-x+\gamma^{2} x}{R^{3}}=\sum_{n=0}^{\infty} H_{n}^{\prime}(x) s^{n} .
$$


Тогда с учетом равенства (26) получим

$$
\begin{aligned}
\frac{\partial G_{0}(x, s)}{\partial x}+\frac{\partial G_{0}(x, s)}{\partial s} & =\frac{\gamma^{2} x}{R^{3}}=\sum_{n=0}^{\infty} H_{n}^{\prime}(x) s^{n}+\sum_{n=0}^{\infty} n H_{n}(x) s^{n-1}= \\
& =\sum_{n=0}^{\infty}\left[(n+1) H_{n+1}(x)+H_{n}^{\prime}(x)\right] s^{n} .
\end{aligned}
$$

Далее, с учетом следствия 6 получим

$$
\frac{\gamma^{2} x}{R^{3}}=\sum_{n=0}^{\infty}(n+1)\left[\left(1+\frac{1}{x^{2}-1}\right) H_{n+1}(x)-\frac{x}{x^{2}-1} H_{n}(x)\right] s^{n},
$$

откуда

$$
\frac{1-x^{2}}{R^{3}}=\frac{1}{\gamma^{2}} \sum_{n=0}^{\infty}(n+1)\left(H_{n}(x)-x H_{n+1}(x)\right) s^{n} .
$$

ТЕорема 7. Порождающая функиия $\left(1-s^{2}\right) / R^{3}$ имеет следующее разложение:

$$
\frac{1-s^{2}}{R^{3}}=\frac{1}{Q}+\frac{1}{\gamma^{2}} \sum_{n=1}^{\infty}\left[(n+1)\left(\gamma^{2}-1\right) x H_{n+1}(x)+\left(n\left(1+\gamma^{2}\right)+1\right) H_{n}(x)\right] s^{n} .
$$

ДокАЗАтЕльство. Искомое разложение получим из равенства

$$
\frac{1-s^{2}}{R^{3}}=\frac{1-x^{2}}{R^{3}}+\frac{x-s}{R^{3}} x+\frac{x-s}{R^{3}} s
$$

и известных разложений для каждого слагаемого в правой части равенства (34). Так как функции $H_{n}(x)$ порождаются функцией $G_{0}(x, s)$, из $(26)$ получим

$$
\frac{x-s}{R^{3}}=\sum_{n=1}^{\infty} n H_{n}(x) s^{n-1}=\sum_{n=0}^{\infty}(n+1) H_{n+1}(x) s^{n} .
$$

Тогда из формул (32) и (34) следует, что

$$
\begin{aligned}
\frac{1-s^{2}}{R^{3}}= & \frac{1}{\gamma^{2}} \sum_{n=0}^{\infty}(n+1)\left(H_{n}(x)-x H_{n+1}(x)\right) s^{n}+ \\
& +\sum_{n=0}^{\infty}(n+1) x H_{n+1}(x) s^{n}+\sum_{n=1}^{\infty} n H_{n}(x) s^{n}= \\
= & \frac{1}{\gamma^{2}} H_{0}(x)+x\left(1-\frac{1}{\gamma^{2}}\right) H_{1}(x)+ \\
& +\sum_{n=1}^{\infty}\left[(n+1) x\left(1-\frac{1}{\gamma^{2}}\right) H_{n+1}(x)+\left(n+\frac{n+1}{\gamma^{2}}\right) H_{n}(x)\right] s^{n} .
\end{aligned}
$$

Нетрудно проверить, что сумма первых двух слагаемых в последнем равенстве равна $1 / Q^{3}$, ввиду того что $H_{0}(x)=1 / Q, H_{1}(x)=x / Q^{3}$ (см. равенство $(20)$ ), откуда окончательно получаем соотношение (33). 
3.3. Функции, ортогональные к многочленам меньшей степени на отрезке $[-1,1]$, можно с помощью замены переменной свести к функциям, ортогональным к многочленам меньшей степени на отрезке $[0,1]$. Рассмотрим одно семейство таких функций, которое имеет приложение в задачах электромагнетизма.

В порождающей функции $G_{1}(x, s)$ произведем замену переменной $x=2 t-1$. Тогда

$$
G_{1}\left(x(t), s\left(s_{1}\right)\right)=\frac{t(1-t)}{2 R_{1}^{3}}
$$

где

$$
R_{1}=R_{1}\left(t, s_{1}\right)=\sqrt{\left(t-s_{1}\right)^{2}+\gamma^{2} t(1-t)}, \quad s_{1}=\frac{1+s}{2} .
$$

Поскольку $G_{1}(x, s)$ - порождающая функция, из теоремы 1 следует, что

$$
\int_{0}^{1} \frac{t(1-t)}{2 R_{1}^{3}}(2 t-1)^{n} d t=p_{n}\left(s_{1}\right)
$$

для любых целых неотрицательных значений $n$. Отсюда следует, что интегралы

$$
\int_{0}^{1} \frac{t(1-t)}{2 R_{1}^{3}} t^{n} d t
$$

также являются многочленами степени $n$ по переменной $s_{1}$. Таким образом, в силу теоремы 1 функция $G_{1}\left(t, s_{1}\right)$ также является порождающей на отрезке $[0,1]$.

Найдем явный вид функций, порождаемых $G_{1}\left(t, s_{1}\right)$. Из соотношений $(32)$ и (35) следует, что

$$
\frac{t(1-t)}{R_{1}^{3}}=\frac{2}{\gamma^{2}} \sum_{n=0}^{\infty}(n+1)\left[H_{n}(t)-(2 t-1) H_{n+1}(t)\right]\left(2 s_{1}-1\right)^{n},
$$

где

$$
H_{n}(t)=\frac{1}{q^{n+1}} P_{n}\left(\frac{2 t-1}{q}\right), \quad q=q(t)=Q(x(t))=\sqrt{(2 t-1)^{2}+4 \gamma^{2} t(1-t)} .
$$

Введем обозначения

$$
q_{1}=q_{1}(t)=R_{1}\left(t, \frac{1}{2}\right)=\sqrt{\left(t-\frac{1}{2}\right)^{2}+\gamma^{2} t(1-t)}, \quad h_{n}(t)=\frac{1}{q_{1}^{n+1}} P_{n}\left(\frac{t-1 / 2}{q_{1}}\right) .
$$

Тогда

$$
q=2 q_{1}, \quad H_{n}(t)=\frac{h_{n}(t)}{2^{n+1}}, \quad \frac{t(1-t)}{R_{1}^{3}}=\sum_{n=0}^{\infty} \tilde{h}_{n}(t)\left(s_{1}-\frac{1}{2}\right)^{n},
$$

где

$$
\tilde{h}_{n}(t)=\frac{n+1}{\gamma^{2}}\left(h_{n}(t)-\left(t-\frac{1}{2}\right) h_{n+1}(t)\right)
$$




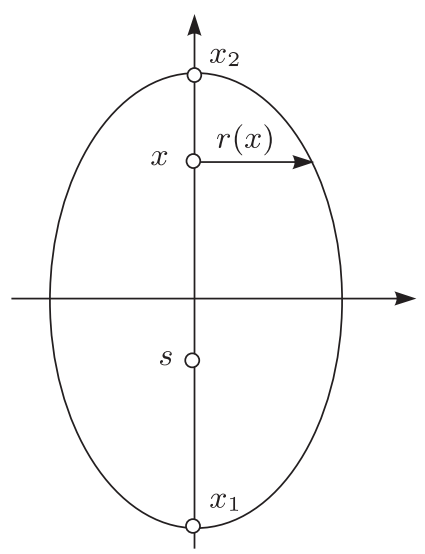

Рис. 1

\section{4. ПРИМЕНЕНИЕ ФУНКЦИЙ, ОРТОГОНАЛЬНЫХ К МНОГОЧЛЕНАМ МЕНЬШЕЙ СТЕПЕНИ, ПРИ РЕШЕНИИ ФИЗИЧЕСКИХ ЗАДАЧ}

Рассмотрим тело, имеющее форму эллипсоида вращения с полуосями, равными 1 и $\gamma, \gamma \leqslant 1$. Обозначим через $r(x)$ радиус поперечного сечения тела на расстоянии $x$ от начала координат, а через $d l(x)$ - элемент длины образующей тела вращения (см. рис. 1). Пусть это тело находится в некоторой среде, обладающей свойством симметрии, причем наибольшая полуось тела направлена вдоль оси симметрии среды.

Решение всех задач, которые будут изложены ниже, сводится к решению интегральных уравнений Фредгольма первого рода с ядрами, являющимися порождающими функциями. Поэтому если правые части этих уравнений суть многочлены степени $N$, то согласно теореме 2 их решения также будут многочленами той же степени, коэффициенты которых могут быть найдены как решение системы линейных уравнений (10). Для определения элементов матрицы $\mathbf{F}$ из (10) мы будем использовать порождаемые функции, которые были получены в разделе 3 . Элементы матриц для всех рассмотренных задач можно выразить через момент или линейную комбинацию двух моментов от многочленов Лежандра с некоторым весом.

4.1. Осесимметричное электрическое поле индуцирует на поверхности проводящего тела, имеющего ту же ось симметрии, что и электрическое поле, осесимметричное распределение поверхностных зарядов $\sigma(x)$. Эти заряды создают в точке $s$ на оси (см. рис. 1) потенциал

$$
\varphi(s)=\frac{1}{2 \varepsilon_{0}} \int_{x_{1}}^{x_{2}} \frac{r(x)}{\sqrt{(x-s)^{2}+r^{2}(x)}} \sigma(x) d l(x)
$$

и напряженность осевого электрического поля поверхностных зарядов

$$
E(s)=-\frac{1}{2 \varepsilon_{0}} \int_{x_{1}}^{x_{2}} \frac{r(x)(x-s)}{\left[(x-s)^{2}+r^{2}(x)\right]^{3 / 2}} \sigma(x) d l(x),
$$


где $\varepsilon_{0}$ - электрическая постоянная. При экранировке электрический потенциал $\varphi(s)$ компенсирует на оси начальный потенциал $\varphi_{0}(s)$ и начальную напряженность $E_{0}(s)$ электрического поля.

Поверхностные заряды создают на оси внутри тела потенциал, равный по величине, но обратный по знаку потенциалу начального поля, и напряженность осевого электрического поля, равную по величине, но обратную по знаку начальной напряженности осевого электрического поля. Отсюда следует, что плотность поверхностного заряда $\sigma(x)$ проводящего тела с нулевым потенциалом для тела, имеющего форму эллипсоида вращения с полуосями, равными 1 и $\gamma$, и помещенного во внешнее осесимметричное поле с потенциалом $\varphi_{0}(s)$ на оси или с осевой напряженностью $E_{0}(s)$, можно определить двумя способами: решив уравнение

$$
\int_{-1}^{1} \frac{1}{\sqrt{(x-s)^{2}+\gamma^{2}\left(1-x^{2}\right)}} \sigma_{0}(x) d x=-\varphi_{0}(s), \quad-1<s<1,
$$

или уравнение

$$
\int_{-1}^{1} \frac{x-s}{\left[(x-s)^{2}+\gamma^{2}\left(1-x^{2}\right)\right]^{3 / 2}} \sigma_{0}(x) d x=E_{0}(s), \quad-1<s<1 .
$$

Тогда

$$
\sigma(x)=\chi_{\mathrm{e}}(x) \sigma_{0}(x), \quad \text { где } \quad \chi_{\mathrm{e}}(x)=2 \varepsilon_{0}\left[r(x) \frac{d l(x)}{d x}\right]^{-1}=\frac{2 \varepsilon_{0}}{\sqrt{1-x^{2}\left(1-\gamma^{2}\right)}} .
$$

В интегральном уравнении (38) ядром является порождающая функция $G_{0}(x, s)($ см. формулу (16)), а порождаемые функции $H_{n}(x)$ определены в (20). Согласно следствиям 3 и 5 в матрице $\mathbf{F}$ системы линейных уравнений (10) равны нулю элементы, стоящие в нечетных верхних диагоналях.

Элементы матрицы $\mathbf{F}$ можно вычислить более удобным образом. Действительно, согласно формулам (11) и (20)

$$
F_{n k}=\int_{-1}^{1} H_{n-1}(x) x^{k-1} d x=\int_{-1}^{1} \frac{1}{Q^{n}} P_{n-1}\left(\frac{x}{Q}\right) x^{k-1} d x,
$$

где $Q=\sqrt{x^{2}\left(1-\gamma^{2}\right)+\gamma^{2}}$. Введем замену переменных $z=x / Q$. Тогда получим $F_{n k}=\gamma^{k-n} L_{n k}$, где

$$
L_{n k}=\int_{-1}^{1} \frac{P_{n-1}(z)}{Q_{0}^{k-n+2}} z^{k-1} d z, \quad Q_{0}=Q_{0}(z)=\sqrt{1-z^{2}\left(1-\gamma^{2}\right)} .
$$

Ненулевые значения интегралов $L_{n k}$ можно выразить через гипергеометрические функции. Положим $n-1=2 m+\delta$, где $\delta$ принимает значения 0 или 1 . Тогда согласно справочнику [6]

$$
\begin{aligned}
L_{2 m+\delta, k}= & \frac{(-1)^{m}((1-k+\delta) / 2)_{m}}{((k+\delta) / 2)_{m+1}} \times \\
& \times{ }_{3} F_{2}\left(\frac{k-\delta+1}{2}-m, \frac{k}{2}, \frac{k+1}{2} ; \frac{k+\delta}{2}+m+1, \frac{k-\delta+1}{2}-m ; 1-\gamma^{2}\right),
\end{aligned}
$$


где $(a)_{m}$ - символ Похгаммера: $(a)_{0}=1,(a)_{m}=\prod_{l=1}^{m}(a+l-1)$. Поскольку первый и пятый параметры в обобщенной гипергеометрической функции ${ }_{3} F_{2}$ совпадают, мы имеем

$$
L_{2 m+\delta, k}=\frac{(-1)^{m}((1-k+\delta) / 2)_{m}}{((k+\delta) / 2)_{m+1}} F\left(\frac{k}{2}, \frac{k+1}{2} ; \frac{k+\delta}{2}+m+1 ; 1-\gamma^{2}\right) .
$$

Перейдем к решению уравнения (39). Ядром этого уравнения является порождающая функция (26), а порождаемыми функциями будут $H_{n}^{(1)}(x)=(n+1) H_{n+1}(x)$ (см. формулу $(7))$. Поскольку четность функций $H_{n}(x)$ совпадает с четностью индекса (см. следствие 5 ), функции $H_{n}^{(1)}(x)$ будут четными при нечетном $n$ и нечетными в противном случае. Тогда $\int_{-1}^{1} H_{n}^{(1)}(x) x^{k} d x=0$, если $n+k$ - четное число, и, в частности, диагональные элементы матрицы $\mathbf{F}$ в (10) будут равны нулю. Чтобы система линейных уравнений (10) с треугольной матрицей и нулевой диагональю была совместна, необходимо выполнение условия $b_{N}=0$. Тогда система линейных уравнений (15) может быть записана как

$$
\sum_{k=1}^{N} c_{k} \int_{-1}^{1} H_{n}^{(1)}(x) x^{k} d x=b_{n}, \quad n=0,1, \ldots, N-1 .
$$

Представим эту систему в матричном виде $\mathbf{F}^{(1)} \mathbf{c}_{1}=\mathbf{b}_{1}$, где

$$
\mathbf{c}_{1}=\left(c_{1}, c_{2}, \ldots, c_{N}\right)^{\mathrm{T}}, \quad \mathbf{b}_{1}=\left(b_{0}, b_{1}, \ldots, b_{N-1}\right)^{\mathrm{T}},
$$

и матричные элементы матрицы $\mathbf{F}^{(1)}$ задаются формулой

$$
F_{n k}^{(1)}=\int_{-1}^{1} n H_{n}(x) x^{k} d x, \quad k=1, \ldots, N, \quad n=0,1, \ldots, N-1 .
$$

Решение уравнения (39) имеет вид

$$
\sigma_{0}(x)=\sum_{k=0}^{N} c_{k} x^{k}
$$

где $c_{0}$ - произвольная постоянная. Наличие произвольной константы в формуле (42) следует также из того, что интеграл от порождающей функции $G_{0}(x, s)$ по $x \in[-1,1]$ равен константе, не зависящей от переменной $s$, и, следовательно, интеграл от функции $\frac{\partial G_{0}(x, s)}{\partial s}$ по этому отрезку равен нулю. Элементы матрицы $\mathbf{F}^{(1)}$, стоящие в нечетных верхних диагоналях, равны нулю (см. раздел 2). Ненулевые элементы матрицы $\mathbf{F}^{(1)}$ определяются согласно соотношениям (20), (40) и (41) по формуле $F_{n k}^{(1)}=n \gamma^{k-n} L_{n+1, k+1}$.

4.2. Осесимметричное начальное магнитное поле индуцирует на поверхности сверхпроводящего тела, имеющего ту же ось симметрии, что и магнитное поле, круговые токи вокруг общей оси, когда тело помещается в поле. Согласно формуле Био-Савара [7] эти токи создают следующее магнитное поле на оси:

$$
B(s)=\frac{\mu_{0}}{2} \int_{x_{1}}^{x_{2}} \frac{r^{2}(x)}{\left[(x-s)^{2}+r^{2}(x)\right]^{3 / 2}} j(x) d l(x), \quad x_{1}<s<x_{2},
$$




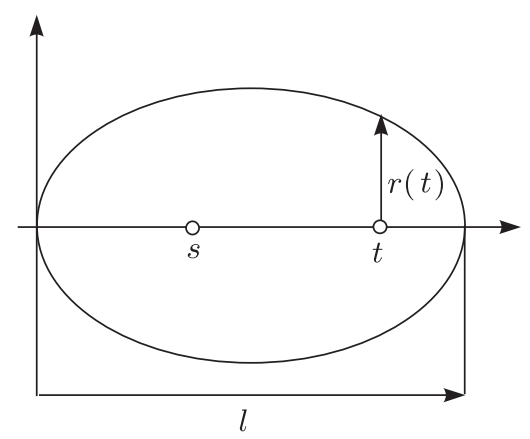

Рис. 2

где $j(x)$ - поверхностная плотность тока, $\mu_{0}$ - магнитная постоянная. Магнитное поле $B(s)$ компенсирует на оси начальное магнитное поле: $B(s)=-B_{0}(s)$. Поверхностную плотность тока $j(x)$ сверхпроводящего эллипсоида вращения можно найти, решив уравнение

$$
\gamma^{2} \int_{-1}^{1} \frac{1-x^{2}}{\left[(x-s)^{2}+\gamma^{2}\left(1-x^{2}\right)\right]^{3 / 2}} j_{0}(x) d x=-\frac{2}{\mu_{0}} B_{0}(s), \quad-1<s<1 .
$$

Тогда

$$
j(x)=\chi_{\mathrm{m}}(x) j_{0}(x), \quad \text { где } \quad \chi_{\mathrm{m}}(x)=\frac{d x}{d l}=\sqrt{\frac{1-x^{2}}{1-x^{2}\left(1-\gamma^{2}\right)}} .
$$

В уравнении (44) ядром является порождающая функция $G_{1}(x, s)$, заданная равенством (30), а порождаемые функции суть $\widetilde{H}_{n}(x)$ из (31). Из формулы (31) и следствия 5 вытекает, что четность функции $\widetilde{H}_{n}(x)$ совпадает с четностью числа $n$. Таким образом, в силу следствия 3 в матрице $\mathbf{F}$ системы линейных уравнений элементы нечетных верхних диагоналей равны нулю. Ненулевые элементы матрицы $\mathbf{F}$ имеют согласно формулам (31) и (40) следующий вид:

$$
F_{n k}=n \gamma^{k-n}\left(L_{n k}-L_{n+1, k+1}\right) .
$$

Если начало координат находится в вершине эллипсоида (см. рис. 2), то уравнение (43) принимает вид

$$
B(s)=\frac{\mu_{0}}{2} \int_{0}^{l} \frac{r^{2}(t)}{\left[(t-s)^{2}+r^{2}(t)\right]^{3 / 2}} j(t) d l(t) .
$$

В этом случае поверхностная плотность тока для эллипсоида вращения с большой полуосью единичной длины определяется из уравнения

$$
\gamma^{2} \int_{0}^{1} \frac{t(1-t)}{\left[(t-s)^{2}+\gamma^{2} t(1-t)\right]^{3 / 2}} j_{0}(t) d t=-\frac{2}{\mu_{0}} B_{0}(s)
$$

Мы имеем

$$
j(t)=j_{0}(t) \frac{d t}{d l}, \quad \frac{d t}{d l}=\sqrt{\frac{t(1-t)}{t(1-t)+\gamma^{2}(t-1 / 2)^{2}}} .
$$


В уравнении (46) ядром является порождающая функция (35). Для определения линейной плотности тока $j_{0}(t)$ необходимо привести правую часть уравнения (46) к виду $\sum_{n=0}^{N} b_{n}(s-1 / 2)^{n}$. В этом случае искомые коэффициенты многочлена также определяются из формулы $(10)$, где порождаемые функции $\tilde{h}_{n}(t)$ заданы в $(37)$. Чтобы элементы нечетных верхних диагоналей в матрице $\mathbf{F}$ системы линейных уравнений, отвечающей уравнению (46), были равны нулю, решение уравнения (46) будем искать в виде суммы: $j_{0}(t)=\sum_{k=0}^{N} c_{k}(t-1 / 2)^{k}$. Такое разложение искомого решения позволит также выразить ненулевые элементы матрицы $\mathbf{F}$ через значения интегралов $L_{n k}$. Действительно, согласно формуле (37)

$$
\begin{aligned}
F_{n k} & =\gamma^{2} \int_{0}^{1} \tilde{h}_{n-1}\left(t-\frac{1}{2}\right)^{k-1} d t= \\
& =n \int_{0}^{1}\left[\frac{1}{q_{1}^{n}} P_{n-1}\left(\frac{t-1 / 2}{q_{1}}\right)-\left(t-\frac{1}{2}\right) \frac{1}{q_{1}^{n+1}} P_{n}\left(\frac{t-1 / 2}{q_{1}}\right)\right]\left(t-\frac{1}{2}\right)^{k-1} d t .
\end{aligned}
$$

Совершим замену переменных $z=(t-1 / 2) / q_{1}$. Тогда получим

$$
F_{n k}=n\left(\frac{\gamma}{2}\right)^{k-n}\left(L_{n k}-L_{n+1, k+1}\right)
$$

4.3. Распределение скоростей в идеальной стационарной струе, обтекающей тело, аналогично распределению магнитного поля вне такого же сверхпроводящего тела. Аналог уравнения (43), определяющего скорость жидкости $v$ вблизи поверхности осесимметричного тела, соосного струе идеальной жидкости, имеет следующий вид [2] (см. рис. 1):

$$
\frac{1}{2} \int_{x_{1}}^{x_{2}} \frac{r^{2}(x)}{\left[(x-s)^{2}+r^{2}(x)\right]^{3 / 2}} u(x) d x=v_{0}(s), \quad x_{1}<s<x_{2} .
$$

где $u(x)=v(x) \frac{d l}{d x}$ и $v_{0}(x)$ - скорость жидкости на оси в отсутствии тела. Пусть тело представляет собой эллипсоид вращения с полуосями 1 и $\gamma$, помещенный вдоль оси струи так, что полуось единичной длины направлена по оси симметрии струи. Тогда из уравнения (47) следует, что

$$
\frac{\gamma^{2}}{2} \int_{-1}^{1} \frac{1-x^{2}}{\left[(x-s)^{2}+\gamma^{2}\left(1-x^{2}\right)\right]^{3 / 2}} u(x) d x=v_{0}(s), \quad-1<s<1,
$$

при этом

$$
v(x)=\sqrt{\frac{1-x^{2}}{1-x^{2}\left(1-\gamma^{2}\right)}} u(x) .
$$

Ненулевые элементы матрицы $\mathbf{F}$, отвечающей уравнению (48), имеют аналогично формуле (45) следующий вид:

$$
F_{n k}=\frac{1}{2} n \gamma^{k-n}\left(L_{n k}-L_{n+1, k+1}\right) .
$$




\section{5. ЗАКЛЮЧЕНИЕ}

Предложенный в настоящей работе подход к решению осесимметричных задач физики, несомненно, не ограничивается рассмотренными задачами и может быть применен для нахождения физических характеристик других осесимметричных тел в средах, обладающих свойством симметрии. Для заряженного шара во внешнем электрическом поле некоторые его физические характеристики можно определить в явном виде [8].

\section{Список литературы}

[1] А. О. Савченко, О. Я. Савченко, ЖТФ, 77:7 (2007), 130-133.

[2] А. О. Савченко, О.Я. Савченко, ТМФ, 170:3 (2012), 381-392.

[3] А. О. Савченко, О.Я. Савченко, Сиб. журн. вычисл. матем., 15:3 (2012), 321-327.

[4] П. К. Суетин, Классические ортогональные многочлены, Наука, М., 1976.

[5] И. С. Градштейн, И. М. Рыжик, Таблицы интегралов, сумм, рядов и произведений, Физматлит, М., 1963.

[6] А.П. Прудников, Ю.А. Брычков, О.И. Маричев, Интегралы и ряды. Специалъные функции, Наука, М., 1983.

[7] J. Orear, Physics, Cornell Univ., New York, 1979.

[8] А. О. Савченко, О.Я. Савченко, Ж. въчисл. матем. и матем. физ., 53:4 (2013), 675-684.

Поступила в редакцию 21.06.2013, после доработки 24.09.2013 